\title{
Cinema e fascismo
}

L'articolo analizza il rapporto tra cinema e fascismo, dall'uso propagandistico della cinematografia durante gli anni del regime alle immagini del passato fascista veicolate nelle pellicole più recenti.

The article analyzes the relationship between cinema and fascism, from the propaganda during the Mussolini's regime to the images of the fascist past conveyed in the most recent movies.

Se il cinema è stato, da subito, utilizzato come strumento di propaganda e di guerra, giocando un ruolo di primo piano nel meccanismo di ricognizione delle linee nemiche già nel corso della Prima guerra mondiale, bisogna dare atto a Benito Mussolini di essere stato il primo dittatore ad avere compreso fino in fondo le potenzialità del nuovo mezzo espressivo in questo senso. Mentre a Mosca si iniziavano gli esperimenti che avrebbero poi condotto alla nascita della cosiddetta "avanguardia sovietica", già all'indomani della Marcia su Roma Mussolini offre mezzi e sostegno politico a coloro che, fra i suoi sostenitori, proponevano un uso sistematico del cinema a scopo propagandistico, creando già nel 1924 quell'Istituto Luce (L'unione cinematografica educativa) promosso da Luciano De Feo e Giacomo Paulucci de' Calboli Barone, il cui scopo era, appunto, contribuire alla formazione del nuovo italiano fascista che avrebbe dominato la scena per il ventennio successivo. Se il cinema, come recita una celebre sentenza mussoliniana, era "l'arma più forte", bisognava che gli investimenti nel suo sviluppo fossero all'altezza della situazione, ed è per questo che la nuova istituzione poté godere di amplissimi mezzi e autonomia, diventando la voce ufficiale della nazione, nonché 
uno dei pilastri della propaganda del Ministero della cultura popolare, rispondendo però, sempre, direttamente al Duce per il proprio operato.

Attraverso i celebri cinegiornali si delineò l'immagine ufficiale dell'Italia imperiale che il regime si proponeva di costruire, nonché le basi della retorica e della iconografia fascista. Una rappresentazione estremamente coesa e coerente, ovviamente estremamente tendenziosa sotto il profilo ideologico e basata in gran parte su un meccanismo di rimozione di ogni problema e ostacolo incontrato dal fascismo. In pratica, il Luce andò a creare per la prima volta un universo mediatico alternativo, somigliante ma sostanzialmente indipendente rispetto alla realtà di un paese che manteneva una serie innumerevole di problemi, specialmente legati a corruzione e arretratezza. Quello del Luce fu quindi un paese immaginario, costruito inizialmente per assecondare il bisogno di consenso del regime e quindi per compiacere quello stesso regime, che finirà per convincersi della veridicità di un tale simulacro e arrivando ad ignorare la fragilità di una nazione ancora lontana dall'aver completato il processo di modernizzazione. Ma il rapporto fra cinema e fascismo non si riduce certamente all'istituzione e al sostegno fornito all'Istituto Luce.

\section{Il fascismo implicito della modernizzazione}

Per capire il complesso e intricato sistema rappresentato dal cinema del Ventennio, tuttavia, bisogna tenere presente due fattori preliminari. Il primo riguarda la condizione disastrosa in cui versava l'industria cinematografica nazionale all'indomani della presa del potere da parte di Benito Mussolini. Infatti, tra il 1908 e il 1915, il cinema italiano aveva ricoperto un ruolo di primissimo piano a livello internazionale, sperimentando strade che avrebbero influenzato lo sviluppo del cinematografo in tutto il mondo. Per fare un paio di esempi, in Italia era stata inaugurata per la prima volta la formula del lungometraggio e, sempre in Italia, era stato messo a punto il fenomeno del divismo che sarebbe diventato uno dei principi fondamentali del cinema americano (il cosiddetto star system). Ancora, l'industria cinematografica nazionale era stata capace di realizzare uno sforzo produttivo imponente come quello che aveva portato alla realizzazione del primo grande kolossal storico-mitologico, Cabiria (1914) di Giovanni Pastrone. Tuttavia, difficoltà economiche legate alla Prima guerra mondiale, speculazioni sbagliate come quella dell'Unione cinematografica italiana (Uci) e l'emergere di un trust a vocazione globale come quello hollywoodiano, avevano gettato la cinematografia nazionale sul lastrico, facendo crollare la produzione e costringendo 
la maggior parte dei registi italiani (da Augusto Genina in giù) ad emigrare in Francia o in Germania.

Questo è lo scenario depresso con cui il regime deve confrontarsi nel tentativo di far rinascere l'industria cinematografica nazionale, un'impresa cui i Mussolini padre e figlio (specialmente Vittorio) si dedicheranno con passione a partire dalla fine degli anni Trenta, con una serie di mosse che passano specialmente attraverso la creazione di varie strutture, fra le quali vanno annoverate:

a) La nascita della Mostra internazionale di arte cinematografica al Lido di Venezia, fortemente voluta dal gerarca veneto Giuseppe Volpi di Misurata, il primo dei grandi festival europei e vetrina fondamentale per far capire l'importanza anche simbolica che il fascismo attribuiva al cinema.

b) La creazione di una grande casa di produzione di stato, basata sui teatri di posa della casa di produzione romana Cines, la cui ricostruzione fu affidata prima al produttore e distributore Stefano Pittaluga, quindi a Ludovico Toepliz e altri. Da qui nascerà, a metà degli anni Trenta, la stessa Cinecittà, grazie agli investimenti a fondo perduto che l'Impero riverserà sul cinema (anche in questo caso, primo nel mondo).

c) La nascita del Centro sperimentale di cinematografia (Csc), la prima grande scuola di cinema italiana, diretta inizialmente da Luigi Chiarini e animata anche da intellettuali che certo non avevano particolare simpatia per il fascismo (ad esempio, Umberto Barbaro). Da lì proviene quella elaborazione teorica che condurrà anche alla nascita del neorealismo, e dal Csc uscirà buona parte di quelle figure autoriali che contribuiranno alla grandezza del cinema italiano nei decenni a venire, da Michelangelo Antonioni a Giuseppe De Santis, fino a Marco Bellocchio e Liliana Cavani.

d) L'istituzione di un grande apparato di censura e controllo sulla produzione cinematografica (ma più in generale sui film che potevano circolare sul territorio nazionale), la Direzione generale per la cinematografia, la cui direzione sarà affidata al giornalista Luigi Freddi, capace di elaborare strategie composite e perversamente acute per la gestione del cinema in Italia.

Infatti, il secondo fattore da tenere presente per comprendere il rapporto fra cinematografo e regime, può essere sintetizzato così: il fascismo è stato una dittatura imperfetta anche per quanto riguarda il cinema. Lo dimostra il fatto che, fino alle leggi protezionistiche emanate dal ministro Alfieri nel 1938, l'Italia resta in larghissima parte un paese colonizzato sotto il profilo dell'offerta cinematografica, in quanto la maggioranza assoluta dei biglietti venduti nel paese erano acquistati per vedere film americani, western, musical, commedie, comici e quant'altro, con percentuali che - a seconda delle annate - si aggiravano fra il 70 e l' $80 \%$. Questo 
fenomeno è la riprova del fatto che la rinascita dell'industria cinematografica nazionale è sempre stata concepita dal regime come un'operazione di immagine e non un'impresa industriale destinata a fare concorrenza a Hollywood neppure sul territorio. Inoltre, appare altrettanto chiaro che per Mussolini il cinema rappresentava anche un grande apparato di "distrazione di massa" e non era quindi il caso di andare a turbare un equilibrio di preferenze e gusti sedimentati che non ostacolava in nulla l'egemonia del fascismo. Tanto più che, se da una parte non si voleva dare all'esterno l'impressione di una dittatura chiusa verso l'esterno, dall'altro lato, come avrà modo di ripetere spesso lo stesso Luigi Freddi, il cinema americano era portatore di valori legati all'eroismo bellico e al trionfo del coraggio e della mascolinità che risultavano perfettamente funzionali anche alla retorica dominante durante il Ventennio.

\section{Il fascismo come rivoluzione e come destino}

Per le stesse ragioni, buona parte della produzione del rinascente cinema italiano durante gli anni Trenta venne dedicata a film di costume ricalcati proprio sui modelli statunitensi, ovviamente riadattati al contesto italiano, come le celebri "commedie dei telefoni bianchi", attraverso cui offrire l'immagine edulcorata di un paese sorridente e ottimista, votato a un nuovo benessere e rapidamente avviato verso l'industrializzazione. È il paese che traspare fra le righe delle trame convenzionali di film come La segretaria privata (di Goffredo Alessandrini, 1931), Gli uomini che mascalzoni (di Mario Camerini, 1932), Il signor Max (sempre di Camerini, 1937) o Maddalena zero in condotta (di Vittorio De Sica, 1940). Ma in questo scenario non potevano ovviamente mancare anche un certo numero di film riguardanti l'avvento e il consolidamento del fascismo, e in particolare la gloria della leadership mussoliniana. In questo senso, se il primo film a celebrare i fasti della Marcia su Roma era stato, già nel 1923, Il grido dell'aquila di Mario Volpi, dieci anni dopo, nel 1933, viene commissionato all'amico personale del duce, Giovacchino Forzano, un film celebrativo che si intitolerà Camicia nera e che sarà girato negli studi di Pisorno (tra Pisa e Livorno) appositamente costruiti per questa impresa. Come anche in Vecchia guardia (1934) di Alessandro Blasetti, la trama ricalca la tesi ufficiale relativa alle ragioni storiche dell'avvento del fascismo, che si sarebbe affermato come antidoto al degrado morale di una borghesia corrotta e al culmine di uno scontro generazionale, dal quale sarebbero uscite vincitrici le forze migliori della gioventù, capaci di sanare la frattura della società civile e scongiurare l'avvento del bolscevismo. 
Questa beatificazione del fascismo come movimento rivoluzionario abile a rinsaldare l'identità della nazione e ad affermare un nuovo ordine, trova il proprio controcanto in opere di rievocazione storica, nelle quali la presa del potere da parte di Mussolini viene inscritta in una prospettiva a dir poco deterministica che porta a una rivalutazione dell'intera storia della penisola. Che si trattasse dell'esercito romano impegnato contro le armate africane di Annibale, come in Scipione l'Africano (di Carmine Gallone, 1937), oppure di un condottiero autoctono che si opponeva ai giochi di palazzo delle corti europee, come in Ettore Fieramosca (di Alessandro Blasetti, 1938) o persino del Garibaldi che prosegue la propria opera di unificazione del paese nonostante le titubanze della casa reale come in 1860 (di Alessandro Blasetti, 1934), c'è sempre un filo conduttore che ricollega le imprese dei grandi uomini del passato con la figura di un condottiero moderno, il cui compito è quello di ricondurre l'Italia al proprio destino imperiale.

\section{Cinema bellico: I'Impero in action e il fascismo dal volto umano}

È dunque inevitabile che, a metà degli anni Trenta, quando ormai sono chiare a tutti le ambizioni politiche di Mussolini, relative alle future alleanze e all'espansone africana dell'Impero, anche il cinema venga mobilitato a sostegno di questo progetto. Ecco allora film come Aldebaran (di Alessandro Blasetti, 1935) o Luciano Serra Pilota (di Goffredo Alessandrini, 1938), nei quali le imprese belliche sono inscritte nella logica del melodramma maschile e l'eroismo militare arriva a riscattare individui che hanno rischiato di compromettere la propria anima per gelosia o per irresponsabilità, grazie al ruolo loro assegnato - rispettivamente dalla Marina o dall'Aviazione. Ancora, ne L'assedio dell'Alcazar (di Augusto Genina, 1940) o in Giarabub (di Goffredo Alessandrini, 1942), ad essere celebrato è l'eroismo incrollabile dei militari italiani, impegnati sul fronte spagnolo in sostegno delle truppe franchiste o in una strenua lotta all'ultimo sangue contro le truppe della "perfida Albione", in pellicole effettivamente ben costruite e ben dirette, secondo la falsariga dell'epica che si può solitamente trovare nel cinema bellico americano.

Tuttavia, questo tipo di film di propaganda bellica, complice l'allentarsi dei controlli corrispondenti all'inizio della Seconda guerra mondiale, diventano il luogo di una paradossale sperimentazione. Infatti, prima Francesco De Robertis con film come Uomini sul fondo (1941) o Alfa Tau! (1942) e quindi Roberto Rossellini con la trilogia composta da La nave bianca (1941), Un pilota ritorna (1942) e L'uomo dalla croce (1943), realizzano altrettanti film propagandistici prodotti dal 
Ministero per la marina militare che presentano però strutture e modi di rappresentazione assolutamente atipici. Si tratta per lo più di film corali, con protagonisti che parlano dialetti vari e sembrano in tutto e per tutto gente presa dalla strada, uomini e donne comuni, lontanissimi dall'ideale dell'eroe predestinato. Figure quotidiane che si trovano a compiere imprese straordinarie quasi loro malgrado, all'interno di film dal ritmo disteso, dove dominano lunghe sequenze di vita quotidiana e non vengono affatto rispettate tutte le convenzioni narrative relative alla suspense tipiche del cinema di genere. Anche per queste ragioni, si è spesso evidenziato il legame genetico che - al di là del nome di Rossellini - questi film intrattengono con il fenomeno destinato a segnare la storia del cinema italiano a partire dall'immediato Dopoguerra, vale a dire il cosiddetto neorealismo cinematografico.

\section{Fascismo neorealista e fascismo come entertainment: il grande tabù}

Eviteremo di richiamare le caratteristiche che hanno reso il neorealismo quasi un sinonimo di cinema della modernità, troppo note e troppo complesse perché ci si debba soffermare su di esse in questa sede, ma è abbastanza ovvio che nel cinema italiano a partire dal 1945 la rappresentazione del fascismo appaia radicalmente cambiata di segno. Se adesso i fascisti sono comprensibilmente diventati i "cattivi", vale la pena sottolineare il fatto che la lettura storica del fenomeno fascista debba comunque rispondere a esigenze politiche di riconciliazione che ne impediscono un corretto inquadramento sotto il profilo storico. Che si tratti della celebre epopea concepita inizialmente da Sergio Amidei e quindi proseguira dal solo Rossellini in Roma Città Aperta (1945) e in Paisà (1946), che si tratti di un documentario collettivo (al quale presero parte figure del calibro di Giuseppe De Santis, Luchino Visconti, Mario Serandrei e molte altre) nato sotto la sponsorizzazione del Partito comunista come Giorni di gloria (1945), o perfino di film di genere di grande impatto popolare, costruiti sul principio narrativo e spettacolare del melodramma lirico, come Avanti a lui tremava tutta Roma (di Carmine Gallone, 1946), il copione storiografico implicito non cambia. Gli italiani diventano a tutti gli effetti vittime sostanzialmente incolpevoli di un'occupazione straniera, portata avanti con la complicità di un risicato manipolo di traditori fascisti.

Come ampiamente dimostrato dallo studioso inglese David Forgacs [2000] in un suo volume dedicato proprio a Roma Città Aperta, anche nell'ambito del neorealismo scompare ogni accenno agli anni del consenso, all'armistizio, alle atrocità compiute dall'esercito italiano durante le guerre coloniali, alla complicità di parte 
della popolazione civile sia nell'applicazione delle leggi razziali sia nei rastrellamenti seguiti alle azioni partigiane. La gloriosa pagina della Resistenza diventa il grande fenomeno palingenetico, capace di lavare tutte le responsabilità storiche della nazione, fino alla Repubblica di Salò (e il fatto che alcuni degli stessi registi più rappresentativi del dopoguerra fossero stati gli stessi della propaganda mussoliniana è forse sufficiente a spiegare questa ansia ecumenica).

Del resto, quando nel 1953 il cineasta e giornalista Renzo Renzi pubblica sulle pagine della rivista "Cinema Nuovo" una proposta per un soggetto intitolato L'armata S'Agapò, nel quale erano contenuti racconti e riflessioni sulla propria esperienza di ufficiale dell'esercito italiano in Grecia, con un ritratto tutt'altro che lusinghiero di un'esperienza che contraddiceva integralmente l'idea degli "italiani brava gente", la reazione dell'Esercito fu immediata. Renzi e lo stesso direttore responsabile della rivista, Guido Aristarco, vennero arrestati nel carcere militare di Peschiera e sottoposti al giudizio di un tribunale militare per vilipendio delle forze armate. Grazie a una vastissima mobilitazione di intellettuali se la cavarono una condanna simbolica, pochi mesi con la condizionale, ma il messaggio che il giudizio del tribunale militare avrebbe potuto comminare in autonomia qualsiasi pena arrivò forte e chiaro e nessuno, per diversi anni, si azzardò più a offrire ritratti negativi delle autorità italiane, anche di quelle del periodo fascista.

Non è un caso che nel corso degli anni Cinquanta si sviluppi un filone bellicoavventuroso, inaugurato dal solito Francesco De Robertis con Uomini e cieli (1947) e proseguito da titoli come Carica Eroica (ancora di De Robertis, 1952), I sette dell'Orsa Maggiore (Duilio Coletti, 1953), Siluri umani (Antonio Leonviola, 1954) o Divisione Folgore (Duilio Coletti, 1954), nei quali l'appartenenza degli eroi alla X Mas o ad altri corpi speciali dell'esercito fascista appare un fatto secondario e contingente, paradossalmente privo di connotazioni politiche in film che sembrano votati al puro intrattenimento e ad una implicita rivalutazione delle forze armate nazionali.

\section{Il tabù infranto e il fascismo (della commedia) all'italiana}

Sarà solo a partire dal 1959, complici il boom economico, il cambiamento dello scenario politico internazionale e il coinvolgimento governativo di forze socialiste, che si potrà ricominciare a svolgere una riflessione sul Ventennio e sul dramma del fascismo e del nazismo. A inaugurare il nuovo clima un film che apparentemente non trattava il tema in modo diretto, essendo dedicato al conflitto precedente. Parliamo di La Grande Guerra (1959) di Mario Monicelli, nel quale i due antieroi interpretati da Vittorio Gassman e Alberto Sordi compiono un atto 
di sacrificio involontario, demitizzando il ruolo dell'esercito. Il successo del film e la mancata reazione delle autorità autorizzano diversi cineasti ad un approccio diretto al tema, infrangendo - talvolta attraverso il pretesto della commedia - il tabù dell'armistizio. È il caso di Tutti a casa (Luigi Comencini, 1960) o de Il federale (Salce, 1961), ma anche di Anni ruggenti (Luigi Zampa, 1962), La marcia su Roma (Dino Risi, 1962), I due marescialli (Sergio Corbucci, 1961) o I due colonnelli (Steno, 1962), interpretati dalla coppia composta da Totò e Vittorio De Sica. Il quale, appena prima, aveva frequentato il tema, nel ruolo di regista, in un film di fortissimo impatto drammatico come La ciociara (1960) e come attore in un dramma pirandelliano come Il generale Della Rovere (Roberto Rossellini, 1959), tratti rispettivamente da due racconti di Moravia e Montanelli. Ma il discorso sul fascismo viene declinato in maniera drammatica anche in una serie di altri titoli nel corso degli stessi anni, da Tiro al piccione (Giuliano Montaldo, 1961) a Il carro armato dell' 8 settembre (Gianni Puccini, 1960), da Estate violenta (Valerio Zurlini, 1959) a Il processo di Verona (Carlo Lizzani, 1962), fino ad esperimenti sofisticati come il film sui campi di sterminio di Gillo Pontecorvo, Kapò (1959) o il documentario a base d'archivio, All'armi siam fascisti (1962) firmato da tre particolarissimi cineasti come Lino Del Fra, Cecilia Mangini e Lino Micciché.

\section{Fascismo d'autore, fascismo pop e riscrittura della storia}

Negli anni seguenti, il rapporto con le molte cicatrici e i traumi di un passato ingombrante e doloroso diventa oggetto di numerose riflessioni compiute da autori diversi, con intenti a volte antitetici che vanno dalla monumentalizzazione dell'antifascismo all'inquadramento storico dei conflitti intergenerazionali, fino alle metafore relative alla deriva della società contemporanea, ciascuno all'interno di un proprio percorso stilistico e della propria poetica. Tra i più attivi, in questo senso, è senz'altro Bernardo Bertolucci, che prima svolge due accorate riflessioni sull'impossibilità di chiudere i conti col passato in due film preziosi e controversi come Il conformista e La strategia del ragno (entrambi del 1970), quindi inscrive il discorso sul fascismo nel quadro di un conflitto ideologico più ampio, sottolineando la relazione fra l'antifascismo e i movimenti rivoluzionari del proletariato in una bizzarra ed epica riproposizione del realismo socialista in chiave hollywoodiana come Novecento (1976).

Altrettanto particolari e personali sono le letture del rapporto fra fascismo e neofascismo della società dei consumi offerta da Pier Paolo Pasolini in Salò o le 120 giornate di Sodoma (1976) o del fascismo come malattia connessa all'infantilismo che definisce una delle dominanti della psicologia degli italiani che ritro- 
viamo in Amarcord (1974) di Federico Fellini. Altri film di alto impegno civile che segnano gli anni Settanta sono Il delitto Matteotti (Florestano Vancini 1973), L'Agnese va a morire (Giuliano Montaldo, 1976) o Mussolini ultimo atto (Carlo Lizzani, 1974), mentre un registro nostalgico e più intimista nella rappresentazione del dramma storico si ritrova in opere come Il giardino dei Finzi Contini (Vittorio De Sica, 1970), Questa specie d'amore (Alberto Bevilacqua, 1972) o Una giornata particolare (Ettore Scola, 1977), scritto da Maurizio Costanzo.

Ma a partire dagli anni Settanta inizia a manifestarsi anche una certa tendenza a declinare in chiave di popular culture, ovvero pop, la rappresentazione del fascismo. Uno dei principali esponenti di questa tendenza è certamente Tinto Brass, che diverse volte si cimenta con la messa in scena del fascismo e della sua estetica, accentuandone al massimo la componente glamour, nel quadro di un cinema dalle forti connotazioni erotiche, al limite della pornografia, come in Salon Kitty (1975), La chiave (1983) o Senso '45 (2002), ma operazioni riconducibili alla cosiddetta "exploitation" del fascismo si ritrovano anche il film come Quando c'era lui... caro lei (Giancarlo Santi, 1978) con Paolo Villaggio, Le vie del signore sono finite (Massimo Troisi, 1987), Mediterraneo (Gabriele Salvatores, 1991), La vita è bella (Roberto Benigni, 1997), El Alamein (Enzo Monteleone, 2002), Le rose del deserto (Mario Monicelli, 2006): film diversissimi fra loro anche rispetto agli esiti qualitativi, film generazionali, di azione, dedicati a perseguire un discorso sul ruolo della poesia, di aneddoti personali e quant'altro, ma accomunati dalla comune tendenza a inscrivere la rappresentazione del fascismo nell'estetica e nei linguaggi tipici della cultura di massa (intesa qui nella sua accezione più generica). Ultimo caso in ordine di tempo, il sensibile In guerra per amore (2016) di Pierfrancesco Diliberto, in arte Pif, che ha utilizzato l'epoca storica dell'occupazione per una più ampia riflessione sulla nascita e la funzione sociale della mafia. In generale, tuttavia, una tendenza particolarmente significativa, nel quadro del cinema contemporaneo che continua a manifestare una spiccata propensione verso le pratiche che caratterizzano la postmodernità, comporta una rappresentazione della storia che ne implica una radicale e libera riscrittura. Se il prototipo di questa attitudine, a livello internazionale, è il film Inglourious Bastards - Bastardi senza gloria (2009) di Quentin Tarantino, dove un manipolo di soldati scelti ebrei riusciva a uccidere Adolf Hitler in una eroica impresa in terra tedesca, anche in Italia si sono avuti esempi assimilabili. Riscritture legate al dibattito ideologico che ha determinato numerose polemiche, come in alcuni film che buona parte della critica ha catalogato come "di destra" (ma stiamo ovviamente semplificando), da Porzus (1997) di Renzo Martinelli a Il sangue dei vinti (2008) di Michele Soavi, ispirato evidentemente alle opere di Gianpaolo Pansa, ma anche 
operazioni che cercano di strappare il discorso sul fascismo e l'antifascismo ai processi di istituzionalizzazione che talvolta tendono a rendere opachi gli aspetti prettamente umani di tali vicende. È quanto ha fatto ad esempio Daniele Luchetti, recuperando il bellissimo libro di Luigi Meneghello I piccoli maestri (1997), ma anche Ettore Scola con Concorrenza sleale (2001), sul rapporto fra popolazione civile e persecuzione degli ebrei nella Roma fascista, o Marco Tullio Giordana in Sanguepazzo (2008), dedicato ad alcune figure divistiche di quella pagina dimenticata della storia del cinema italiano, il cosiddetto "cinema di Salò". Ma i tentativi forse più riusciti e radicali sono stati quelli compiuti da Marco Bellocchio con Vincere (2009) e Giorgio Diritti con L'uomo che verrà (2010). In entrambi i casi, vicende storiche ampiamente indagate, come l'internamento in manicomio della compagna (Irene Dlaser) e del figlio naturale di Mussolini o l'eccidio di Marzabotto, sono oggetto di una narrazione filmica che trascende ogni pretesa di verosimiglianza e rispetto del dato storico in quanto tale per fare di queste storie una più ampia riflessione sulla natura umana e sul rapporto con la memoria collettiva.

\section{Bibliografia}

Forgacs D. 2000, Rome Open City, London: British Film Institute

Ricci S. 2008, Cinema and Fascism, Berkeley: University of California Press

Venturini A. 2015, La politica cinematografica del regime fascista, Roma: Carocci

Zagarrio V. 2004, Cinema e fascismo. Film, modelli, immaginari, Venezia: Marsilio

\section{Risorse online}

Video della relazione al convegno Narrare il fascismo (Predappio, 20-21 gennaio 2017) https://www.youtube.com/watch?v=WEzBdnr6hYk 